\title{
LA EMIGRACIÓN CUBANA HACIA LOS ESTADOS UNIDOS, PARTE 1, DE 1860 A 1989: UN ANÁLISIS ESTADÍSTICO Y COMPARATIVO
}

\section{Salim Lamrani}

Université de La Réunion

\begin{abstract}
Salim Lamrani es Profesor de historia de América Latina en la Universidad de La Reunión, especialista de las relaciones entre Cuba y los Estados Unidos. Ha publicado The Economic War Against Cuba. A Historical and Legal Perspective of the U.S. Blockade (New York, Monthly Review Press, 2013) y Cuba, the Media and the Challenge of Impartiality (New York, Monthly Review Press, 2015). En total, es autor de diez libros publicados en francés, inglés, español e italiano.
\end{abstract}

\section{Resumen}

En los Estados Unidos en particular, pero también en los países occidentales, desde 1959 la emigración cubana es objeto de dos consensos. Primero, la Revolución cubana habría ocasionado un importante movimiento migratorio hacia los Estados Unidos. Luego, éste sería el reflejo del rechazo del proceso de transformación social estructurado en torno a un sistema político con partido único elaborado por Fidel Castro. Las estadísticas migratorias de las autoridades estadounidenses disponibles para el período que va de 1860 a 2019, permitirán arrojar una luz sobre esta cuestión al comparar los distintos flujos procedentes de Cuba en diferentes épocas (1860-1959, 1960-1989, 1990-2019). Del mismo modo, un análisis comparativo de las corrientes migratorias emitidas por los países de la región evaluará la pertinencia de estas dos hipótesis.

Palabras claves: Cuba, Estados Unidos, emigración, América, estadísticas, análisis comparativo 


\section{Introducción}

Desde la Revolución Cubana de 1959, la problemática migratoria es objeto de debates. Dos postulados parecen hacer consenso. Primero, es comúnmente aceptado que el proceso de transformación social emprendido bajo el liderazgo de Fidel Castro desde su llegada al poder y la instauración de un sistema político con partido único, han estado en el origen de un fenómeno migratorio sin precedente en la historia del país, principalmente hacia Estados Unidos. Segundo, una parte importante de los observadores de la realidad cubana considera que la emigración masiva de cubanos a partir de 1959 refleja la expresión del rechazo del sistema político, económico y social actualmente vigente en la mayor isla del Caribe.

Las estadísticas disponibles relativas a la emigración cubana hacia los Estados Unidos -el período que va de 1860 a 2019 con la excepción de los años 1900-1920permiten evaluar la pertinencia de estas parrillas de lectura ${ }^{1}$. Existen debates sobre la exactitud de las distintas fuentes. Por eso los datos usados en este estudio son las cifras oficiales de los servicios de inmigración de los Estados Unidos. ¿Cuál era la realidad migratoria en Cuba antes de 1959 con respecto al resto del continente americano? ¿Acaso la emigración cubana a partir de 1959 es excepcional en comparación con las cifras anteriores y las salidas procedentes de los otros países de la región? ¿Existen factores objetivos específicos de incitación a la emigración cubana hacia los Estados Unidos a partir de 1959? ¿Cuál fue la realidad migratoria durante el "Período Especial" en general y más precisamente durante la famosa crisis de los Balseros en 1994? ¿Cuál es la realidad de los flujos migratorios hoy día? Finalmente, ¿acaso se puede considerar la emigración cubana como un instrumento de evaluación de la adhesión de la población al régimen político y socioeconómico presente en la Isla?

Después de una presentación del postulado inicial, tres ejes cronológicos estructurarán este estudio. En un primer tiempo, convendrá analizar la emigración cubana durante el período 1860-1959, es decir desde el inicio de la década que marcó el desencadenamiento de la Primera Guerra de Independencia en Cuba (1868-1878) hasta el fin del reinado del régimen militar de Fulgencio Batista en 1959. En un segundo tiempo, se echará una mirada a la realidad migratoria cubana de 1960 hasta 1989, o sea desde la imposición de las primeras sanciones económicas por parte de la administración Eisenhower hasta la caída del Muro de Berlín. Finalmente, este análisis se concluirá con la realidad migratoria de Cuba desde el fin de la Guerra Fría hasta hoy día (1990-2019).

1 Este artículo abarca un período largo desde un punto de vista puramente estadístico. El objetivo no es desarrollar las motivaciones de las distintas olas migratorias. 


\section{Presentación del postulado inicial}

Según los Estados Unidos, la emigración cubana hacia su territorio desde 1959 es un hecho que hay que interpretar bajo un ángulo político. Este fenómeno sería la ilustración del rechazo que suscita entre una parte sustancial de la población el sistema político y socioeconómico vigente en la Isla, lo que habría llevado a muchos cubanos a tomar el camino del exilio. Así, según el Departamento de Estado, «en los años posteriores a la Revolución de 1959, cientos de miles de cubanos han huido de la isla». (Department of State 2000) Washington subraya que este fenómeno migratorio perdura hasta el día de hoy: «Desde hace décadas, los cubanos han huido de Cuba, a menudo por barco, en busca de la libertad en Estados Unidos» (United States Accounting Office 1995).

El demógrafo Gérard-François Dumont, profesor en Sorbonne Université, comparte esta opinión. Según él, la emigración cubana hacia los Estados Unidos desde 1959 se explica esencialmente por razones políticas:

En Cuba el déficit migratorio acumulado se establece, para el período 1959-2015, a más de un millón, o sea el $12 \%$ de la población media durante este período. En menos de medio siglo [sic], un cubano de cado ocho eligió entonces abandonar la Isla y darle la espalda a la Revolución castrista. Los cuatro quintos optan por los Estados Unidos que, en los períodos en que este país los acepta, acogen a estos inmigrantes como "refugiados", víctimas de la dictadura comunista. [.. .] Semejante intensidad de éxodo se explica principalmente por el carácter liberticida del régimen castrista. [. . . S Se encuentra facilitada ya que Castro no desea de ningún modo impedir la emigración de eventuales opositores (Dumont 2015).

El Profesor Félix Masud-Piloto, historiador en la Universidad DePaul de Illinois, ofrece una mirada más matizada y subraya en su obra de referencia From Welcomed Exiles to Illegal Immigrants: Cuban Migration to the U.S., 1959-1995 que «la emigración cubana fue desatada principalmente por cambios revolucionarios complejos en Cuba y por la respuesta de los Estados Unidos a esos cambios, que incluyó, entre otras medidas, favorecer esta emigración». El académico explica los dos motivos de semejante política: «Desestabilizar al Gobierno de Castro privándolo de recursos humanos vitales (tales como los médicos, los profesores y los técnicos) y desprestigiar al régimen alentando la huida de miles de personas de una dictadura comunista hacia una democracia capitalista». Washington ha dedicado importantes recursos a esta política y "ha asumido una responsabilidad financiera a largo plazo para permitir que los cubanos se instalen» en el territorio estadounidense (Masud-Piloto 1996: 2). 
Así, a título de ejemplo, de los 6286 médicos que tenía la Isla en 1959 para una población total de 6 millones de habitantes - o sea una ratio de un doctor para 1064 personas - en el espacio de tres años, 1402 de ellos optaron por abandonar el país e irse a los Estados Unidos atraídos por mejores oportunidades profesionales y una política migratoria favorable elaborada por Washington para los cuadros cubanos de todos los sectores (Rojas Ochoa 2015).

La prensa considera también comúnmente este postulado como una realidad. Así, según la Agence France Presse, «más de dos millones de cubanos han emigrado desde hace medio siglo, de una población hoy día de 11,2 millones de habitantes. Cerca del 80\% de ellos se han marchado a los Estados Unidos, particularmente a la Florida, a 150 kilómetros al norte de la isla» (Agence France Presse 2017).

Conviene confrontar estas distintas parrillas de lectura a la realidad de las estadísticas migratorias.

\section{La emigración cubana hacia Estados Unidos de 1860 a 1959}

\section{0-1869}

Según las estadísticas oficiales del US Department of Homeland Security, de 1860 a 1869, 3420 cubanos optaron por emigrar a los Estados Unidos. La Isla, situada a 150 kilómetros de la Florida y entonces poblada por 1,2 millones de habitantes, siempre ha considerado este país como una tierra de asilo natural a la vez por razones históricas y geográficas. A título de comparación México, mastodonte demográfico (7,7 millones de habitantes) que comparte una inmensa frontera con el poderoso vecino, solo registró 1957 salidas durante este mismo período. Haití, (900.000 habitantes), situado al Este de la Isla, solo registró 78 candidatos a la emigración hacia el norte. En cuanto a Jamaica, ubicada en el sur de Cuba, solo observó 61 salidas. Toda América del Sur (18 millones de habitantes), desde Colombia hasta Argentina, originó 1536 salidas hacia los Estados Unidos, o sea dos veces menos que la Isla. Así, Cuba produjo una emisión migratoria durante esa década 106 veces superior a la de un gigante como Brasil (7,2 millones /32 salidas). En cuanto a América Central (1,7 millones), solo observó 70 partidas (US Department of Homeland Security 2020: table 2).

\section{0-1879}

De 1870 a 1879 , período marcado por la Primera Guerra de Independencia (1868-1878), Cuba emitió 8705 salidas hacia el territorio estadounidense, o sea un aumento del $150 \%$ con respecto a la década anterior. Por su parte, México generó una producción migratoria de 5133 personas en el mismo período. Haití observó 
149 salidas y Jamaica 257. América del Sur, por su lado, solo registró 1109 partidas, o sea cerca de ocho veces menos que la Isla. A título de ejemplo, Brasil tuvo un flujo migratorio de 219 personas, Colombia (2,2 millones) de 196, Venezuela (1,5 millones) de 190 y Perú (1,9 millones) de 127. En cuanto a América Central, solo observó 202 partidas (US Department of Homeland Security 2020: table 2).

\section{0-1889}

De 1880 a 1889 Cuba registró un total de 20134 salidas, o sea un alza de alrededor del $130 \%$ con respecto al decenio precedente. Por su parte, México vio su cifra migratoria caer a 2 045. Haití, por su lado, emitió 255 salidas, mientras que Jamaica vio emigrar a 355 personas hacia los Estados Unidos. América Central observó una emigración total de 359 personas, durante el mismo período, mientras que América del Sur produjo 1954 salidas. Colombia fue el principal proveedor de capital humano en esta parte del mundo con 1210 salidas (U.S. Department of Homeland Security, 2020: table 2).

\section{0-1899}

En la década 1890-1899, marcada por la Segunda Guerra de Independencia (1895-1898) y el fin del dominio colonial español, la emigración cubana hacia los Estados Unidos siguió creciendo con un total de 23669 salidas, o sea un alza del $17 \%$. Por su parte, México vio su flujo migratorio bajar a solo 734 elementos, o sea treinta y dos veces menos que la Isla. Haití (101) y Jamaica (223) observaron a su vez menos salidas que los años anteriores. Esta tendencia también se dio en América Central, con un total de 674 partidas, y en América del Sur con 1389 salidas (US Department of Homeland Security 2020: table 2).

Clasificación por número de emigrantes hacia los Estados Unidos de 1860 a 1899

\begin{tabular}{|c|c|c|c|c|c|c|c|c|}
\hline & \multirow[t]{2}{*}{$\begin{array}{l}\text { Países y } \\
\text { regiones }\end{array}$} & \multicolumn{2}{|c|}{$\begin{array}{l}\text { Población en } \\
\text { millones de } \\
\text { habitantes }\end{array}$} & \multirow[t]{2}{*}{$\begin{array}{l}1860- \\
1869\end{array}$} & \multirow[t]{2}{*}{$\begin{array}{l}1870- \\
1879\end{array}$} & \multirow[t]{2}{*}{$\begin{array}{l}1880- \\
1889\end{array}$} & \multirow[t]{2}{*}{$\begin{array}{l}1890- \\
1899\end{array}$} & \multirow[t]{2}{*}{$\begin{array}{l}1860- \\
1899\end{array}$} \\
\hline & & 1850 & 1900 & & & & & \\
\hline 1 & Cuba & 1,2 & 1,6 & 3420 & 8705 & 20134 & 23669 & 55928 \\
\hline 2 & México & 7,7 & 13,6 & 1957 & 5133 & 2405 & 734 & 10229 \\
\hline 3 & América del Sur & 18 & 39,2 & 1536 & 1109 & 1954 & 1389 & 5988 \\
\hline 4 & América Central & 1,7 & 3,5 & 70 & 202 & 359 & 674 & 1305 \\
\hline
\end{tabular}

Fuentes: U.S. Department of Homeland Security, 2020: table 2; Brea, 2003. 
Así, durante este período de cuarenta años, Cuba ocupó el primer puesto de la región (México, América Central, Caribe y América del Sur) en términos de emisión migratoria hacia los Estados Unidos, delante de un gigante demográfico que comparte una frontera común con la tierra de destino como México, y delante de otro coloso demográfico, pero más alejado, como Brasil. Esta realidad muestra que Cuba fue históricamente una tierra de emigración hacia el vecino del norte, mucho más que cualquier otra nación de la zona que va de Río Bravo a Tierra del Fuego.

\section{0-1959}

Las estadísticas oficiales del Gobierno de los Estados Unidos no están disponibles para Cuba del período que va de 1900 a 1920. Conviene ahora interesarse en la década 1920-1929. Cuba era entonces una República formalmente independiente, pero que se encontraba en realidad bajo el dominio de Washington, ya que los Estados Unidos habían impuesto la Enmienda Platt a la Isla desde 1902. Con respecto al último decenio del siglo XIX, Cuba redujo su emisión migratoria, con solo 12769 salidas hacia el norte. En cambio, México vio su emigración crecer de modo exponencial con no menos de 498945 partidas, o sea un aumento del $269 \%$ con respecto a la década anterior. Por su parte, América Central observó una emisión migratoria de 16511 personas y América del Sur de 43025 personas. A pesar de esta baja, Cuba envió tres veces más emigrantes hacia los Estados Unidos que un país como Brasil (4 627), nación que produjo la emisión migratoria más fuerte de América Latina, después de México y la Isla. De 1920 a 1929, Cuba ocupó el segundo puesto de la clasificación detrás de México (US Department of Homeland Security 2020: table 2).

Para el período que va de 1930 a 1939, la emigración cubana hacia el norte siguió bajando con 10641 salidas. Por su parte, México dividió por quince su emisión migratoria hacia los Estados Unidos, con solo 32709 partidas, probablemente a causa de las consecuencias de la crisis de 1929. Los vecinos de Cuba tuvieron una producción migratoria menos fuerte. Así, República Dominicana, cuyas estadísticas aparecen por primera vez en este decenio, vio a 1161 de sus ciudadanos emigrar hacia el vecino del norte. Haití solo observó 207 salidas. Toda América Central emitió 6840 migrantes. En cuanto a América del Sur, el número de migrantes bajó a 9 900. En esta década Cuba conservó su segundo puesto detrás de México (US Department of Homeland Security 2020: table 2).

En cambio, la década 1940-1949 vio la emigración cubana explotar con respecto a los años anteriores con no menos de 25976 salidas, o sea un auge del 144\%, superando así en cifras absolutas el récord del siglo XIX. Esta cifra es superior a la de todas las otras naciones del Caribe juntas (20 218 emigrantes), a la de América Central (20 135) y a la de América del Sur (19 662). Por su parte México duplicó 
casi su emisión migratoria con 56158 salidas. Otra vez la Isla se clasificó en el segundo puesto del continente (US Department of Homeland Security 2020: table 2).

De 1950 a 1959, la emigración cubana hacia los Estados Unidos alcanzó 73221 salidas, o sea un alza del $181 \%$ con respecto a la década anterior. Por su parte, con 273847 salidas, México observó un aumento del 387\%. República Dominicana superó las 10000 partidas (10 219), o sea un crecimiento del 112\%. Cuba superó así a América Central (40 201) pero se colocó por primera vez detrás América del Sur $(78$ 418) (US Department of Homeland Security 2020: table 2). El Profesor Félix Masud-Piloto precisa que 26527 cubanos abandonaron la Isla en 1959, después de la llegada de Fidel Castro al poder (Masud Piloto 1996: xxvi). Así, de 1950 a 1958, 46694 personas se marcharon de Cuba para los Estados Unidos. Incluso con esta reducción, Cuba conservó su segundo puesto en la clasificación.

Clasificación por número de emigrantes hacia los Estados Unidos de 1920 a 1959

\begin{tabular}{|c|c|c|c|c|c|c|c|c|}
\hline & $\begin{array}{l}\text { Países y } \\
\text { regiones }\end{array}$ & $\begin{array}{l}\text { Población en } \\
\text { millones de } \\
\text { habitantes }\end{array}$ & $\begin{array}{l}1920- \\
1929\end{array}$ & $\begin{array}{l}1930- \\
1939\end{array}$ & $\begin{array}{l}1940- \\
1949\end{array}$ & $\begin{array}{l}1950- \\
1959\end{array}$ & 1959 & Total \\
\hline 1 & México & 27,7 & 498945 & 32709 & 56158 & 273847 & & 861659 \\
\hline 2 & América del Sur & 109,4 & 43025 & 9990 & 19662 & 78418 & & 151095 \\
\hline 3 & Cuba & 5,9 & 12769 & 10641 & 25976 & 73221 & 26527 & 122607 \\
\hline 4 & América Central & 9,3 & 16511 & 6840 & 20135 & 40201 & & 83687 \\
\hline
\end{tabular}

Fuentes: US Department of Homeland Security 2020: table 2; Brea, 2003.

Así, durante el período de cuarenta años que va de 1920 a 1959, en términos de número de salidas hacia los Estados Unidos, Cuba pasó del primer puesto del continente desde México hasta Argentina, al segundo puesto. La Isla produjo una emisión migratoria más fuerte que la de América Central durante las cuatro décadas. También superó a América del Sur salvo en los períodos de 1920 a 1929 y de 1950 a 1959. Cuba conservó así su perfil de nación de emigración, principalmente con destino a los Estados Unidos.

\section{La emigración cubana hacia los Estados Unidos de 1960 a 1989}

\section{El contexto histórico}

Antes de abordar la cuestión migratoria conviene recordar el contexto histórico de la década 1960-1969. En 1959, la Revolución Cubana liderada por Fidel Castro llegó al poder y emprendió un proceso de transformación social radical, 
que puso en tela de juicio el orden establecido, las estructuras socioeconómicas existentes y los privilegios de clase. La recuperación de los recursos naturales del país mediante nacionalizaciones generó fuertes tensiones con la administración Eisenhower, que se opuso resueltamente al nuevo régimen, creando desde los primeros meses una situación conflictiva. En marzo de 1960 la Casa Blanca tomó la decisión formal de derrocar al Gobierno de Osvaldo Dorticós y del Primer Ministro Fidel Castro y lanzó una operación de guerra clandestina contra la Isla con una campaña de terrorismo y sabotaje, creando un sentimiento de inseguridad entre la población (Eisenhower 1960: 850-1).

En julio y octubre de 1960 Washington impuso sanciones económicas contra la Isla suprimiendo primero la cuota azucarera, principal fuente de ingresos del país y poniendo luego término a todas las exportaciones e importaciones entre las dos naciones (Paarlberg 1960: 979-80; Goodpaster 1960: 1089-90). En enero de 1961 los Estados Unidos decidieron romper las relaciones diplomáticas con La Habana (Hurwich 1961: documento 7). En abril de 1961 un contingente paramilitar de cerca de 1500 hombres entrenados y armados por la CIA invadió Bahía de Cochinos, operación destinada a derrocar al poder revolucionario que desembocó en un fracaso patente en el espacio de 72 horas (Kennedy 1961: documento 157). En octubre de 1962, mientras la Casa Blanca proseguía con su política hostil contra Cuba multiplicando los actos de agresión de todo tipo, estalló la Crisis de Octubre, que puso al mundo al borde de un cataclismo nuclear. Después de trece días de tensiones Washington y Moscú encontraron finalmente una solución para un retiro del arsenal nuclear de la Isla (Khrushchev 1962: documento 102).

Así, durante los primeros años de la Revolución Cubana, la Isla vivió en un ambiente de permanentes tensiones marcado por la hostilidad del poderoso vecino y las consecuencias de los actos de agresión económica y paramilitar. Se puede considerar que este contexto geopolítico inaudito constituyó un factor objetivo de incitación a la emigración para una parte de la población.

Del lado de los Estados Unidos, la administración de John F. Kennedy desplegó grandes recursos con la creación en febrero de 1961 del Programa para Refugiados Cubanos (Cuban Refugee Program) del cual se podía beneficiar toda persona de "nacionalidad cubana" que «dejó Cuba el 1 de enero de 1959 o después» (Mitchell 1962). Como indica el texto oficial, esta política gubernamental no tenía precedentes:

Por primera vez en su historia los Estados Unidos se han convertido en el primer asilo para un número importante de personas desplazadas, ya que miles de refugiados cubanos han encontrado refugio político aquí. Por primera vez también, el Gobierno de los Estados Unidos ha juzgado necesario desarrollar un programa para ayudar a los refugiados de otra nación de este hemisferio. (Mitchell 1962) 
Según su director John F. Thomas, «el Cuban Refugee Program es único en el sentido de que se desarrolló a través de la utilización de los servicios de agencias ya existentes en la medida más amplia posible, aunque la responsabilidad general era del Departamento de Salud, Educación y Seguridad Social» (Thomas).

El Programa tenía como objetivo subvenir las necesidades de los emigrantes cubanos proporcionándoles toda la asistencia necesaria. La meta política e ideológica era importante: demostrar la superioridad del capitalismo sobre el comunismo. Este programa se detallaba en nueve puntos:

1. Proporcionar toda la asistencia posible a las agencias de apoyo que brindan diariamente una ayuda a numerosos refugiados, para permitir la instalación del número más grande y asegurarles un empleo.

2. Obtener la asistencia de las agencias a la vez privadas y gubernamentales para ofrecer oportunidades de trabajo concretas a los cubanos desplazados, en acuerdo con la situación general del trabajo en Florida.

3. Proporcionar fondos adicionales para la reubicación de los refugiados en otras regiones, incluso el transporte y los costos de ajustes para las nuevas comunidades y para su eventual regreso a Miami con vistas a una repatriación hacia su país cuando ello sea posible.

4. Proporcionar una asistencia financiera para responder a las necesidades básicas de las familias de refugiados cubanos en dificultad en la región de Miami y en las comunidades de reubicación, administrada a través de los canales local, federal y del Estado y basada en los estándares vigentes en las comunidades involucradas.

5. Proporcionar una asistencia médica básica a través del programa de asistencia financiera que brindan los servicios de salud pública y los servicios de salud infantil, así como los otros servicios necesarios.

6. Proporcionar una asistencia federal para los gastos de escolarización en las escuelas públicas vinculados al impacto no previsto de los niños cubanos refugiados en los establecimientos escolares locales.

7. Elaborar las medidas necesarias para aumentar las oportunidades de formación y de educación para los refugiados cubanos, incluso los médicos, los profesores y los que tienen otra trayectoria profesional.

8. Proporcionar una ayuda financiera para la atención y la protección de los niños no acompañados - el grupo más frágil y más vulnerable entre la población de refugiados.

9. Elaborar un programa de distribución alimentaria, el cual será gestionado por los servicios sociales locales, para que una ayuda alimentaria suplementaria sea distribuida a los refugiados necesitados. (Mitchell 1962) 
Ningún otro grupo nacional se ha beneficiado de un programa de semejante magnitud. A ello se agrega la adopción de la Ley de Ajuste Cubano en 1966, otra legislación de excepción.

\section{La Ley de Ajuste Cubano}

El 2 de noviembre de 1966, de conformidad con la política hostil del Presidente Lyndon B. Johnson, el Congreso de los Estados Unidos decidió adoptar la Ley de Ajuste Cubano - todavía vigente hoy día - que otorga un estatus especial a todos los cubanos que se marchan del país a partir del 1 de enero de 1959. Esta legislación, única en el mundo, permite a todo ciudadano cubano que emigre legal o ilegalmente a los Estados Unidos conseguir al cabo de un año y un día el estatus de residente permanente, así como distintas ayudas sociales. Se puede considerar que este texto constituye objetivamente una herramienta de incitación a la emigración legal e ilegal. He aquí un extracto:

Para ajustar el estatus de los refugiados cubanos y hacerlos residentes legales permanentes de los Estados Unidos, y para otros motivos, [...] el estatus de todo extranjero nativo o ciudadano de Cuba y que fue tramitado y admitido o que se encuentra en libertad condicional en los Estados Unidos después del 1ro de enero de 1959, y que ha estado físicamente presente en los Estados Unidos durante al menos dos años [un año y un día], puede ser ajustado por el Ministro de Justicia [. . . ] como extranjero legalmente admitido para una residencia permanente. [. . .] Las provisiones de esta Ley se aplican a la pareja y a los hijos de todo extranjero descrito en esta subsección, sin consideración de su ciudadanía y lugar de nacimiento, que viven con el extranjero en cuestión en los Estados Unidos. (United States Congress 1966)

Ricardo Alarcón de Quesada, Presidente del Parlamento Cubano de 1993 a 2013, subrayó el carácter específico de la Ley de Ajuste Cubano:

Hay dos elementos fundamentales en esta legislación. Primero, hay una referencia a una fecha muy precisa y todo el mundo sabe lo que evoca. ¿Qué significa el hecho de que aceptan a gente a partir de esa fecha?

Ello significa que excluyen a todos los que llegaron allí antes, y se trataba del segundo país productor de emigrantes hacia los Estados Unidos. Esta ley no concierne a los que llegaron antes del 1ro de enero de 1959, y entonces no pueden beneficiarse de ésa.

¿Por qué están excluidas las personas de origen cubano que llegaron a los Estados Unidos antes de esa fecha? Simplemente porque representaban una población muy importante; si no, no habría ninguna razón para fijar una fecha. 
[Luego], esta ley tiene una dimensión desestabilizadora pues es el instrumento de una política que busca a promover la emigración de los cubanos hacia los Estados Unidos.

La única categoría de personas entre todos los habitantes del planeta que dispone del privilegio de poder adquirir la residencia legal en los Estados Unidos, si se presenta a las autoridades, es la de origen cubano.

El único país en el mundo que dispone de esta ley que estimula, promueve y busca con mucha publicidad y campañas mediáticas y por otras formas de incitación a provocar la emigración de cubanos, únicamente de cubanos, son los Estados Unidos.

Hubo otros programas de incitación y de asistencia tal como el Programa para los Refugiados Cubanos, que ofrecía viviendas y empleos a los emigrantes, contrariamente a lo que se reserva a todo otro inmigrante que, en general, se encuentra abandonado a su suerte cuando tiene un estatus legal y que es encarcelado cuanto está en situación irregular. Ellos no disponen de estos privilegios. (Lamrani 2007: 48-9)

\section{0-1989}

Tomando en cuenta estos elementos, ¿cuál fue la realidad migratoria de Cuba durante los primeros treinta años de la Revolución? Aquí está en cifras.

De 1960 a 1969, no menos de 202030 cubanos dejaron la Isla para ir a los Estados Unidos, o sea un alza del $175 \%$ con respecto a la década anterior. A título de comparación, hubo 83522 salidas de República Dominicana, o sea un aumento de $717 \%$ sobre el mismo período. Del mismo modo, 28992 haitianos emigraron hacia el norte, o sea un crecimiento del 665\%. Con 62218 partidas Jamaica observó un crecimiento del $741 \%$. Este auge migratorio no se limitó a los países del Caribe, ya que concernió a casi todo el continente. Así, América Central produjo una emigración de 98569 personas, o sea un crecimiento del 145\%. En el detalle, Costa Rica, con 17975 salidas, observó un aumento del $344 \%$, Belice (4 185) del 269\%, Guatemala (14 357) del 242\%, Honduras (15 087) del 183\% y El Salvador (4 185) del 182\% (US Department of Homeland Security 2020: table 2).

Por su lado América del Sur emitió 250754 salidas, o sea un alza del 219\%. En el detalle, Argentina vio la partida de 49384 personas, o sea un crecimiento de $202 \%$. Con 6205 candidatos a la emigración, Bolivia observó un aumento del 124\%. Por su parte Brasil, con 29238 salidas, vio su emigración hacia los Estados Unidos crecer en un $153 \%$. En cuanto a Chile (12 384), sus partidas aumentaron en un 165\%. Pasó lo mismo en Colombia (68 371, +339\%), Ecuador (34 107, 
+297\%), Perú (19 783, +230\%), Uruguay (4 089, +298\%) o Venezuela (20 758, $+109 \%$ ) (US Department of Homeland Security 2020: table 2).

Número de emigrantes hacia los Estados Unidos de 1960 a 1969

\begin{tabular}{|c|c|c|c|c|c|}
\hline & Países & $\begin{array}{l}\text { Población en } \\
\text { millones de } \\
\text { habitantes } \\
\text { en } 1960\end{array}$ & $\begin{array}{l}\text { Número de } \\
\text { emigrantes } \\
\text { en la década } \\
1960-1969\end{array}$ & $\begin{array}{l}\text { Evolución } \\
\text { con respecto } \\
\text { a la década } \\
\text { anterior }\end{array}$ & $\begin{array}{l}\text { Clasificación } \\
\text { en porcentaje }\end{array}$ \\
\hline 1 & México & 36,9 & 441824 & $+61 \%$ & 19 \\
\hline 2 & Cuba & 7 & 202030 & $+175 \%$ & 14 \\
\hline 3 & República Dominicana & 3,2 & 83522 & $+717 \%$ & 2 \\
\hline 4 & Colombia & 16,9 & 68371 & $+339 \%$ & 5 \\
\hline 5 & Jamaica & 1,6 & 62218 & $+741 \%$ & 1 \\
\hline 6 & Argentina & 20,6 & 49384 & $+202 \%$ & 11 \\
\hline 7 & Ecuador & 4,4 & 34107 & $+297 \%$ & 7 \\
\hline 8 & Brasil & 72,8 & 29238 & $+153 \%$ & 16 \\
\hline 9 & Haití & 3,8 & 28992 & $+665 \%$ & 3 \\
\hline 10 & Venezuela & 7,6 & 20758 & $+109 \%$ & 18 \\
\hline 11 & Perú & 9,9 & 19783 & $+230 \%$ & 10 \\
\hline 12 & Costa Rica & 1,2 & 17975 & $+344 \%$ & 4 \\
\hline 13 & Honduras & 1,9 & 15087 & $+183 \%$ & 12 \\
\hline 14 & Guatemala & 4 & 14357 & $+242 \%$ & 9 \\
\hline 15 & Chile & 7,6 & 12384 & $+165 \%$ & 15 \\
\hline 16 & Bolivia & 3,4 & 6205 & $+124 \%$ & 17 \\
\hline 17 & Salvador & 2,6 & 4185 & $+182 \%$ & 13 \\
\hline 18 & Belice & 0,09 & 4185 & $+269 \%$ & 8 \\
\hline \multirow[t]{3}{*}{19} & Uruguay & 2,5 & 4089 & $+298 \%$ & 6 \\
\hline & América del Sur & & 250754 & & \\
\hline & América Central & & 98569 & & \\
\hline
\end{tabular}

Fuentes: US Department of Homeland Security 2020: table 2; Brea 2003; World Bank 2020a, b, c.

A finales de los años 1960 Cuba ocupaba todavía el segundo puesto detrás de México (441 824) en términos de salidas migratorias hacia los Estados Unidos, a pesar del Cuban Refugee Program, de la Ley de Ajuste Cubano, las sanciones económicas y la guerra clandestina llevada contra la Isla. En términos de aumento en porcentaje con respecto a la década precedente, la mayor Isla solo ocupaba el decimocuarto puesto del continente latinoamericano. 


\section{0-1979}

En el período que va de 1970 a 1979, 256497 cubanos abandonaron el país hacia los Estados Unidos, o sea un alza del $26 \%$ con respecto a la década anterior. México, con 621218 salidas, observó un crecimiento del 40\%. Por su parte, República Dominicana produjo a 139249 migrantes, o sea un aumento del 66\%. Haití vio la salida de 55192 de sus ciudadanos hacia los Estados Unidos, o sea un crecimiento del 90\%. Jamaica (130 226) vio un alza del 109\%. En lo que se refiere a América Central, con 120376 partidas, la emigración aumentó en un $22 \%$. En el detalle, Belice (6 747) observó un alza del $61 \%$, Guatemala (23 837) del 65\% y el Salvador (29 428) del 104\%. En América del Sur (273 529) la emigración creció de modo más modesto, con un alza del 9\% con respecto a la década anterior. Algunos países como Perú $(25311,+27 \%)$, Ecuador $(47464,+39 \%)$, Uruguay $(8416,+105 \%)$ o Guyana $(38278,+742 \%)$ siguieron la misma tendencia creciente (US Department of Homeland Security 2020: table 2).

Número de emigrantes hacia los Estados Unidos de 1970 a 1979

\begin{tabular}{llcccc}
\hline Países & $\begin{array}{l}\text { Población en } \\
\text { millones de } \\
\text { habitantes } \\
\text { en } 1970\end{array}$ & $\begin{array}{l}\text { Número de } \\
\text { emigrantes } \\
\text { en la década } \\
1970-1979\end{array}$ & $\begin{array}{l}\text { Evolución } \\
\text { con respecto } \\
\text { a la década } \\
\text { anterior }\end{array}$ & $\begin{array}{l}\text { Clasificación } \\
\text { en porcentaje }\end{array}$ \\
\hline 1 & México & 50,6 & 621218 & $+40 \%$ & 9 \\
2 & Cuba & 8,5 & 256497 & $+26 \%$ & 12 \\
3 & República Dominicana & 4,4 & 139249 & $+66 \%$ & 6 \\
4 & Jamaica & 1,87 & 130226 & $+109 \%$ & 2 \\
5 & Haití & 4,5 & 55192 & $+90 \%$ & 5 \\
6 & Ecuador & 6 & 47464 & $+39 \%$ & 10 \\
7 & Guyana & 0,7 & 38278 & $+742 \%$ & 1 \\
8 & Salvador & 3,6 & 29428 & $+104 \%$ & 4 \\
9 & Perú & 13,2 & 25311 & $+27 \%$ & 11 \\
10 & Guatemala & 5,2 & 23837 & $+65 \%$ & 7 \\
11 & Uruguay & 2,8 & 8416 & $+105 \%$ & 3 \\
12 & Belice & 0,11 & 6747 & $+61 \%$ & 8 \\
& América del Sur & & 273529 & & \\
\hline
\end{tabular}

Fuentes: US Department of Homeland Security 2020: table 2; Brea 2003; World Bank 2020a, b, c. 
A finales de los años 1970, Cuba conservaba su segundo puesto en la tabla detrás de México. En términos de porcentaje de alza con respecto a la década anterior, la Isla clasificaba en el duodécimo puesto en el hemisferio.

\section{0-1989}

En 1980, en un contexto de tensiones políticas con los Estados Unidos y de insatisfacciones materiales dentro de la sociedad cubana, sobrevino la crisis de Mariel que desembocó en la salida de alrededor de 125000 cubanos. En abril de 1980, un grupo de seis cubanos penetró por la fuerza en la embajada de Perú. Durante un tiroteo de respuesta hecho por la policía para impedir que el autobús destruyera el portal del recinto diplomático, un agente recibió una bala perdida, que rebotó en el vehículo y perdió la vida. El Gobierno cubano exigió entonces que los organizadores de la acción fueran entregados a las autoridades, lo que se negó a hacer el embajador peruano. La Habana decidió entonces retirar a su personal de seguridad a la representación diplomática, ocasionando una invasión de la embajada por varios miles de candidatos a la emigración (Copeland 1983: 1338-50).

Al inicio los Estados Unidos no estaban implicados en este asunto, que concernía únicamente Cuba y a Perú. El Presidente James Carter, que no obstante había adoptado una política constructiva hacia la Isla desde su llegada al poder, se dejó convencer por su asesor de seguridad nacional, Zbigniew Brzezinski, que lo alentó a tomar una posición sobre el tema. La Casa Blanca declaró entonces que los Estados Unidos abrirían sus puertas a todos los candidatos a la emigración. Éstos serían recibidos “con los brazos abiertos”, declaró el Presidente (Weisman 1980: 1-13).

El Gobierno de La Habana replicó entonces anunciando que todas las personas que deseaban abandonar la Isla hacia los Estados Unidos podrían hacerlo desde el puerto de Mariel, durante un período de seis meses, y autorizó incluso a los cubanos de la Florida a venir a buscar a sus familiares. Según ciertas afirmaciones, las autoridades habrían incluso aprovechado la situación para vaciar las prisiones del país. Así, entre abril y septiembre de 1980, alrededor de 125 000 cubanos abandonaron el país (Hufker 1990: 321-35). Los historiadores y académicos consideran este episodio como una de las mayores olas migratorias que ha conocido Cuba (García 2018; Barquet 1989: 345-56; Aguirre y al. 1997: 487-507; Card 1990: 245-57).

Las estadísticas migratorias oficiales del Gobierno de los Estados Unidos sobre la década 1980-1989 permiten echar una mirada más matizada sobre este período. En efecto, Cuba observó la salida de 132552 personas, o sea una disminución del 93\% con respecto a la década anterior. En el mismo tiempo, 
México vio su emigración crecer al punto de superar el millón con 1009586 partidas, o sea un aumento del 62\%. Por su parte, República Dominicana vio el número de salidas explotar y superar por primera vez el de Cuba con 221552 migraciones, o sea un crecimiento del 59\%. Jamaica también superó a Cuba con 193874 salidas, o sea un crecimiento del 48\%. El Salvador, víctima de una guerra civil, también superó el número de emigrantes que salieron de Cuba con 137418 partidas, o sea un alza del 366\% (US Department of Homeland Security 2020: table 2).

Otros países, sin superar Cuba en cifras absolutas, vieron su emigración aumentar de modo sustancial. Así Haití, con 121406 salidas, observó un crecimiento del $120 \%$. América Central, en su conjunto, vio su número de emigrantes (339 376) crecer en un 181\%. Panamá observó 32957 salidas, o sea un alza del 54\%, Costa Rica (25 017) del 101\%, Belice (14 964) del 121\%, Guatemala (58 847 ) del 151\%, Honduras (39 071) del 149\% y Nicaragua (31 102) del 185\% (US Department of Homeland Security 2020: table 2).

En América del Sur el número de candidatos a la emigración (399 803) aumentó en un 46\% con respecto a la década anterior. Así, Colombia (105 494) observó un alza del 48\%. Por su parte, Brasil (22 944) tuvo un aumento del 23\%, Chile (19 749) del 31\%, Bolivia (9 798) del 73\%, Perú (49 958) del 97\%, Venezuela (22 405) del 103\%, Guyana (85 886) del 124\% y Paraguay (3 518) del $136 \%$. Así, en cifras absolutas, Cuba se clasificó en el quinto puesto del continente durante la década. En términos de porcentaje con respecto al decenio anterior, Cuba solo ocupó el vigésimo puesto de la región (US Department of Homeland Security 2020).

Número de emigrantes hacia los Estados Unidos de 1980 a 1989

\begin{tabular}{llcccc}
\hline Países & $\begin{array}{l}\text { Población en } \\
\text { millones de } \\
\text { habitantes en } \\
1980\end{array}$ & $\begin{array}{l}\text { Número de } \\
\text { emigrantes } \\
\text { en la década } \\
1980-1989\end{array}$ & $\begin{array}{l}\text { Evolución } \\
\text { con respecto } \\
\text { a la década } \\
\text { anterior }\end{array}$ & $\begin{array}{l}\text { Clasificación } \\
\text { en porcentaje }\end{array}$ \\
\hline 1 & México & 67,6 & 1009586 & $+62 \%$ & 13 \\
2 & República Dominicana & 5,7 & 221552 & $+59 \%$ & 14 \\
3 & Jamaica & 2,1 & 193874 & $+48 \%$ & 16 \\
4 & Salvador & 4,6 & 137418 & $+336 \%$ & 16 \\
5 & Cuba & 9,7 & 132552 & $-93 \%$ & 20 \\
6 & Haití & 5,5 & 121406 & $+120 \%$ & 8 \\
7 & Colombia & 28,4 & 105494 & $+48 \%$ & 17 \\
8 & Guyana & 0,78 & 85886 & $+124 \%$ & 6 \\
9 & Guatemala & 6,8 & 58847 & $+151 \%$ & 3
\end{tabular}




$\begin{array}{llrrrr}10 & \text { Perú } & 17,3 & 49958 & +97 \% & 11 \\ 11 & \text { Honduras } & 3,6 & 39071 & +141 \% & 4 \\ 12 & \text { Panamá } & 2 & 32957 & +54 \% & 15 \\ 13 & \text { Nicaragua } & 2,9 & 31102 & +185 \% & 2 \\ 14 & \text { Costa Rica } & 2,3 & 25017 & +101 \% & 10 \\ 15 & \text { Brasil } & 121,7 & 22944 & +23 \% & 19 \\ 16 & \text { Venezuela } & 15,1 & 22405 & +103 \% & 9 \\ 17 & \text { Chile } & 11,1 & 19749 & +31 \% & 18 \\ 18 & \text { Belice } & 0,14 & 14964 & +121 \% & 7 \\ 19 & \text { Bolivia } & 5,4 & 9798 & +73 \% & 12 \\ 20 & \text { Paraguay } & 3,1 & 3518 & +136 \% & 5 \\ & \text { América Central } & & 339376 & & \\ & \text { América del Sur } & & 399803 & & \end{array}$

Fuentes: US Department of Homeland Security 2020: table 2; Brea 2003; World Bank 2020a, b, c.

Así, en el espacio de treinta años, pese a las sanciones económicas, al Cuban Medical Program, a la guerra asimétrica llevada por Washington contra la Isla y a la Ley de Ajuste Cubano, que constituyen elementos objetivos de incitación a la emigración, Cuba pasó del segundo puesto de los países emisores de emigrantes hacia los Estados Unidos en 1960 al quinto en 1989. Salvo México, todos los demás países situados delante de Cuba en la clasificación, disponen de una población inferior a la de la Isla. Así, sobre este período, los cubanos emigraron, en promedio, menos hacia los Estados Unidos que sus vecinos de continente.

\section{Bibliografía}

Agence France Presse (2017) «L'émigration cubaine, un demi-siècle de contentieux”, 13 de enero. https://www.lepoint.fr/monde/l-emigration-cubaine-un-demi-siecle-de-contentieux-13-01-2017-2096799_24.php (sitio consultado el 4 de noviembre de 2020).

Aguirre, B. E., Sáenz, Rogelio, y Sinclair James, Brian (1997) “Marielitos Ten Years Later: The Scarface Legacy", Social Science Quarterly, 78(2): 487-507.

Barquet, Jesús J. (1989) “Clés pour comprendre une génération”, Revue française d'études américaines, 41:345-56.

Brea, Jorge A. (2003) "Population dynamics in Latin America", Population Bulletin (Washington, DC: Population Reference Bureau)volume, 58(1): 7.

Card, David (1990) "The impact for the Mariel boatlift on the Miami labor market", Industrial and Labor Relations Review, 43(2): 245-57.

Copeland, Ronald (1983), "The Cuban boatlift of 1980: Strategies in federal crisis management”, Annals of American Academy of Political and Social Science, 467: 138-50. 
Department of State (2000) “Migration", 16 de marzo. https://1997-2001.state.gov/ regions/wha/cuba/migration.html (sitio consultado el 4 de noviembre de 2020).

Dumont, Gérard-François (2015) “Cuba: Histoire d'un exode", Population et Avenir, 5(725). https://www.cairn.info/revue-population-et-avenir-2015-5-page-3.htm (sitio consultado el 4 de noviembre de 2020).

Eisenhower, Dwight D. (1960) "Paper prepared by the 5412 Committee”, 16 de marzo, Eisenhower Library, Project “Clean Up” Records, Cuba. Secret. Eyes Only. Foreign Relations of the United States, 1958-1960.

García, José Manuel (2018) Voices from Mariel: Oral Histories of the 1980 Cuban Boatlift. Gainesville, FL: University Press of Florida.

Goodpaster, Andrew Jackson (1960) "Memorandum of a conference with the President, White House, Washington”, 17 de octubre, Eisenhower Library, Whitman File, Miscellaneous Material. Secret. Foreign Relations of the United States, 1958-1960.

Hufker, Brian, y Cavender, Gray (1990) "From freedom flotilla to America's burden: The social construction of the Mariel immigrants", Sociological Quarterly, 31(2): 321-35.

Hurwich, Robert A. (1961) "Telegram from the Department of State to the Embassy in Cuba”, 3 de enero, Department of State, Central Files, 611.37/1-361. Unclassified, NIACT, Verbatim Text. Foreign Relations of the United States 1961-1963, Cuba, 1961-1962, documento ${ }^{\circ} 7$.

Kennedy, Robert F. (1961) "Memorandum from the Attorney General (Kennedy) to President Kennedy”, 19 de abril, Kennedy Library, President's Office Files, Countries Series, Cuba, General, April 1961. Foreign Relations of the United States 1961-1963, Cuba, 1961-1962, documento $\mathrm{n}^{\circ} 157$.

Khrushchev, Nikita (1962) "Message from Chairman Khruschev to President Kennedy", 28 de octubre, Department of State, S/S Files, Lot 65 D 438, Miscellaneous. Foreign Relations of the United States, 1961-1963, Volume XI, Cuban Missile Crisis and Aftermath, documento $\mathrm{n}^{\circ} 102$.

Lamrani, Salim (2007) Fidel Castro, Cuba et les Etats-Unis. Pantin: Le Temps des Cerises. Masud-Piloto, Felix (1996) From Welcomed Exiles to Illegal Immigrants: Cuban Migration to the U.S., 1959-1995. Oxford: Rowman \& Littlefield Publishers.

Mitchell, William (1962) “The Cuban Refugee Program”, Social Security Bulletin (marzo): 1-3. https://www.ssa.gov/policy/docs/ssb/v25n3/v25n3p3.pdf (sitio consultado el 4 de noviembre de 2020).

Paarlberg, Don (1960) "Memorandum of discussion, White House”, 6 de julio, Eisenhower Library, Project "Clean Up" Records, Cuba. Foreign Relations of the United States, 1958-1960.

Rojas Ochoa, Francisco (2015) “El número de médicos en Cuba”, Revista Cubana de Salud Pública (enero-marzo). http://scielo.sld.cu/scielo.php?script=sci_arttext\&pi $\mathrm{d}=$ S0864-34662015000100013 (sitio consultado el 23 de marzo de 2020).

Thomas, John F. (sin fecha) "Cuban refugees in the United States". http://www.latinamericanstudies.org/exile/refugees.pdf (sitio consultado el 10 de noviembre de 2020).

United States Congress (1966) “Cuban Adjustment Act”, Public Law 89-732, 2 de noviembre. https://www.govinfo.gov/content/pkg/STATUTE-80/pdf/STATUTE-80-Pg1161. pdf (sitio consultado el 2 de noviembre de 2020). 
United States Department of Homeland Security (2020) "Yearbook of Immigration Statistics 2019", table 2. https://www.dhs.gov/immigration-statistics/yearbook/2019\#" (sitio consultado el 2 de noviembre de 2020).

United States General Accounting Office (1995) “Cuba. U.S. response to the 1994 Cuban migration crisis", septiembre. https://www.gao.gov/assets/230/221797.pdf (sitio consultado el 11 de noviembre de 2020).

Weisman, Steven R. (1980) "President says U.S. offers 'open arms' to Cuban refugees”, New York Times, 6 de mayo.

World Bank (2020a) "Population, total. Belize, 1960-2019". https://data.worldbank.org/ indicator/SP.POP.TOTL ?end=2019\&locations=BZ\&start=1960 (site consulté le 24 novembre 2020).

World Bank (2020b), "Population, total. Guyana, 1960-2019”. https://data.worldbank. org/indicator/SP.POP.TOTL? end=2019\&locations=GY\&start=1960 (site consulté le 23 novembre 2020).

World Bank (2020c), "Population, total. Jamaica, 1960-2019”. https://data.worldbank. org/indicator/SP.POP.TOTL? end=2019\&locations=JM\&start=1960 (sitio consultado el 23 de noviembre de 2020). 